\title{
Acortamiento del primer período del parto con el uso de "Dilatenca"
}

\author{
(El 3-orto, toloxi-1, 2-Propanodiol en el trabajo del parto) ${ }^{1)}$
}

\section{Doctor AlFredo BURgos RIVERA}

El objeto del presente estudio fue acortar tiempo del trabajo del parto, evitar a la parturienta un largo sufrimiento y disminuir el traumatismo sobre el feto.

Todos sabemos, que si se abrevia el primer período, se hace un gran beneficio a la madre, siempre y cuando que con los medios para lograrlo no sobrevengan consecuencias indeseables, tanto para ella como para su hijo.

Por estos motivos nos propusimos hacer el presente estudio en la Clínica Primero de Mayo, del Instituto Colombiano de Seguros Sociales de Bogotá; después de haber presenciado las demostraciones de los delegados cubanos, en la Conferencia de Obstetricia y Ginecología, celebrada en Panamá en el mes de febrero de 1956.

Para ello usamos una droga, cuya fórmula: 3-orto, toloxi-1, 2-propanodiol (dilatenca), tiene la virtud de romper, por bloqueo interneuronas, el círculo vicioso "doldr-espasmo muscular", y que, por su escaso efecto sobre la corteza cerebral, cuanco se usa en dosis terapéuticas es llamada a prestar grandes servicios en la práctica obstétrica.

El método, cuidadosamente estudiado por la escuela cubaria, fue también objeto de cuidadosas investigaciones por parte nuestra, en los ensayos realizados en la mencionada clínica.

(1) Trabajo de tesis considerado como meritorio en la Facultad de Medicina de la Universidad Javeriana; y facilitado a esta redacción para su resumen y revisión por el profesor doctor Santiago Lleras Czdazzi. M. A. F. B. 
En el presente estudio se observaron tanto primigestantes como multigestantes, con el fin de estudiar el efecto de la droga en unas y otras, y poder concluír sobre el beneficio obtenido en la mujer grávida en general. Sin embargo, la preferencia fue por las primíparas, debido a que en ellas el trabajo del parto es más prolongado pudiendo observar mejor los resultados.

Sin embargo, con el fin de tener un amplio conocimiento sobre los efectos, no se seleccionaron las pacientes, pudiéndose cobservar en la casuística casos de desproporción pélvico-fetal, que fueron sometidos a prueba de trabajo y terminaron en cesárea.

Con el objeto de comprobar la bondad de la droga, en ciertos casos nos apartamos del método original en algunas de sus partes; la ruptura de membranas, el empleo de ocitócicos, e inclusive la práctica de la amniotomía sin aplicar la droga, fueron prácticas adicionales; obteniendo así un estudio comparativo de gran valor.

\section{Material y método}

En todos los casos e inicialmente el obstetra tomó y siguió el gobierno y la dirección del parto. Una vez elegido el caso, la paciente fue sometida a un previo examen clínico y obstétrico, reconociendo así el estado del cuello y la evolución del trabajo.

Como condiciones cervicales se requirió: que el cuello estuviera borrado y con dilatación no menor de 2 centímetros; igualmente la comprobación de una buena dinámica uterina fue la regla, pues es de lógica suponer, si queremos abreviar el tiempo de la duración del parto, proporcionando una fácil y rápida dilatación, una dinámica útil constituye su complemento. Siguiendo a la escuela cubana, recomendamos por haberlo utilizado, la estimulación con ocitócicos del miometrio (salvo excepciones).

Con este fin usamos el Pitocín por vía intradérmica.

En seguida se practicó la ruptura de la bolsa de las aguas o amniotomía. Luego la administración oral de la droga, 3-orto, toloxi-1, 2-propanodiol (comercialmente denominada "Dilatenca").

Para la medicación se siguió un método claro y preciso dividiéndola en tres fases.

Primera fase.-1 gramo de Dilatenca (2 tabletas de 0.50 gramos), por vía oral, con cuello borrado y 2 centímetros de dilatación. 
Segunda fase.--Si al cabo de una hora de la primera toma, con buena dinámica uterina, la dilatación cervical no era completa o casi completa, se administró la segunda dosis, que consistía en 1 gramo más de Dilatenca (2 tabletas de 0,50) por vía cral.

Tercera fase.-Teniendo en cuenta lo anterior, y después de hora y media de la segunda dosis (casos en los cuales no había aún dilatación completa), se administró una tercera dosis de 1 gramo (2 tabletas de 0,50 ), en la misma forma por vía oral.

\section{Farmacologia y Farmacodinamia}

La droga que nos ocupa (que en adelante denominaremos como O. T. P. abreviatura convencional de 3-orto, toloxi-1, 2-propanodiol), es un "polvo untuoso", incolorv, inodoro, de sabor ligeramente amargo; produce anestesia de la lengua, se disuelve en cien partes de agua, en diez de éter, en "giicol propilénico y en alcohol”. Su punto de fusión es de 70 a 71 grados centigrados.

$\mathrm{Su}$ toxicidad es rápidamente neutralizada en el organismo $y$ no tiene efectos acumulativos.

Experiencias realizadas por investigadores en perros, usando dosis altas, demostraron que una gran parte de la droga es excretada como compuesto conjugado por vía intestinal, y solo una pequeña parte se elimina por la orina.

Según Kaphan, seguramente es en el hígado donde la droga es detoxificada, por conjugación con el ácido glucurónico y por cxidación parcial. En general, en dosis terapéuticas y por vía oral es inocua, aun cuando se usen dosis relativamente altas.

Algunos investigadores han observado que produce: anorexia, náuseas y más raramente vértigos, que desaparecen disminuyendo la dosis. Nosotros no encontramos tales efectos secundarios en ninguna de nuestras pacientes.

Shoartz reportó no haber encontrado anormalidad funcional de hígado, riñón, ni sistema hematopoyético en investigaciones realizadas con gran número de pacientes a quienes se les había administrado la droga a dosis altas y por tiempo prolongado.

En Inglaterra y el Canadá se ha usado en solución al $10 \%$ en propilenglicol y alcohol, por vía intravenosa, como auxiliar 
en anestesia, habiéndose reportado flebitis, hemolisis y a veces hemoglobinuria. Por este motivo juzgamos peligrosa su adminis¿ración por vía endovenosa en tal concentración. Cuando se ha usado en solución al $2 \%$ y por vía intravenosa, ha sido para conseguir efecto rápido en convulsiones de tipo tetánico. Sin embargo, su empleo repetido, produce nistagmus horizontal y vertical, sensación de calor, sensación de pinchazos, ligera caída de la presión sistólica, inyección conjuntival, visión borrosa, sequecad de la boca, euforia, ligera incoordinación muscular, somnolencia y a veces hemolisis.

El O. T. P. ejerce su acción sobre el sistema nervioso central, cbrando específicamente sobre las neuronas internunciales o de conexión del cordón medular.

Los nervios del útero se derivan principalmente del sistema nervioso simpático, y accesoriamente de los sistemas cerebroespinal y parasimpático; éste representado en cada lado por el nervio pélvico, está formado por algunas fibras derivadas del segundo, tercero y cuarto nervios sacros, que se pierden en el ganglio de Frankenhauser. El sistema simpático llega a la pelvis por intermedio del plejo hipogástrico, que parte de la bifurcación del plejo aórtico, inmediatamente abajo del promontorio, y luego de descender por cada lado, se pierde también en el plejo de Frankenhauser. Dicho plejo está formado por ganglios de diversos tamaños; pero particularmente por una amplia placa ganglionar situada a cada lado del cuello, inmediatamente arriba del fondo de saco posterior y por delante del recto. Las ramas cferentes se dirigen al útero, a la vejiga y a la parte superior de la vagina, y están formadas de fibras mielínicas y amielínicas; algunas de ellas acaban en terminaciones libres entre las fibras musculares, mientras que otrais se sitúan en la superficie del endometrio.

El útero tiene, pues, varias fuentes de inervación: cerebroespinal, vegetativa autónoma, simpático vagal e intrauterina autónoma (placa de Frankenhauser). La doble inervación medular y simpática es a la vez motriz e inhibidora sobre las fibras circulares y longitudinales. Por lo tanto el O. T. P., actuando sobre el centro medular simpático, paraliza la contractilidad del cuello y cumenta la del cuerpo, reforzando el tono muscular.

Apoyando este modo de actuar del O. T. P. están las teorías čel doctor G. R. Givell sobre el mecanismo neuro-muscular uterino. 
"El útero está constituído por fibras musculares lisas, dispuestas en dos sentidos: unas, longitudinales, predominan a nivel del fondo, donde se entrecruzan, pasando de un lado a otro y descendiendo hasta el nivel del istmo por delante, y un poco más abajo, por detrás; otras, circulares, predominan a nivel del tercio inferior del cuerpo, siendo mucho más escasas en el fonả. El cuello está formado exclusivamente por fibras circulares, pero su abundancia es diversamente apreciada por los distintos autores, llegando algunos a negar la presencia de fibras musculares a nivel del "hocico de tenca"; y otros afirman sobre su existencia aunque en poca cantidad (Acconi, Shmidt, Danforth, Hofmeir, Stiere, Novak y Duhrsen).

La desigual dirección de las fibras nos hace pensar que si todas se contraen al mismo tiempo, el parto no puede progresar. Las longitudinales, al acortar su longitud en la contracción, tiran del cuello en toda su circunferencia, y tienden a abrirlo, ya que por su otro extremo se encuentran fijas en el fondo uterino; las circulares, a su vez, al contraerse, cierran el cuello; concluyéndose que un sistema se opone al otro. Para la progresión del parto es, pues, indispensable que el grupo longitudinal predomine sobre el circular. $\mathrm{Y}$ para que esto se realice, es necesario una acción antagónica de ambos grupos musculares, lo que presupone también una inervación antagónica como en otros órganos huecos, estómago, etc.

Se sostiene la teoría que el sistema colinérgico estimula el cuerpo inhibiendo el cuello, mientras que el sistema adrenérgico actúa inversamente.

Es, sin embargo, importante el hecho que las interrelaciones entre ambas ramas del sistema neuro-vegetativo son sumamente estrechas.

Por último, es indispensable saber que la droga en estudio no alcanza a afectar las funciones voluntarias de la paciente, de manera que ésta se mantiene perfectamente consciente, y el mécico puede contar con su valiosa colaboración, hasta el extremo de poderse emplear la psicoterapia, bajo la acción de la droga.

\section{RESULTADOS}

De las sesenta y un historias seguidas en la Clínica Primero de Mayo de Bogotá, cincuenta y una corresponden a pacientes a quienes se les administró la droga; todas ellas por vía oral. 
El resto, o sean diez casos, se les dejó a manera de control, utilizando para la aceleración del primer periodo únicamente la amniotomía.

De acuerdo con el cuadro de resultados tenemos: que treinta de los casos corresponden a primigestantes, entre las cuales aparecen tres casos intervenidos por cesárea, e incluídos en la estadística para valorar los resultados de duración del primer período y no los del total, del parto mismo. De las primigrávidas el menor tiempo transcurrido entre los 2 centímetros, momento de administración de la droga y la dilatación completa, fue de 25 minutos; el más demorado fue de 6 horas (360 minutos) con un promedio de 2 horas 34 minutos (154 minutos). En las 21 multigestantes el menor tiempo que se obtuvo fue de 25 minutos, y el mayor de 3 horas con 10 minutos (190 minutos); con un promedio de 53 minutos, entre los dos centímetros y la dilatación completa. De los diez casos en los cuales únicamente se utilizó ia amniotomía, tenemos 3 casos de primigestantes; 18 horas 40 minutos, entre la ruptura de las membranas y dilatación completa, el más demorado; y siete horas el más rápido; con un promedio de 11 horas 200 minutos. $Y$ siete multigestantes, entre çuienes el período de dilatación más demorado es de 11 horas, y el más rápido de 2 horas 40 minutos; con un promedio de 5 horas 28 minutos.

Como fácilmente se puede ver, la reducción en tiempo del período de dilatación de los casos en los que se utilizó O. T. P., $\in n$ relación con los que se les practicó solamente la amniotomía, es notable. 


\section{CUADRO NUMERO 1 - RESUMEN DE RESULTADOS}

\begin{tabular}{|c|c|c|c|c|c|c|}
\hline $\begin{array}{l}\text { Historia } \\
\text { número }\end{array}$ & Paridad & Edad & V. de $P$. & $\begin{array}{l}\mathrm{T} \text {. de } \mathrm{T} \text {. } \\
\text { antes } \mathrm{M} \text {. }\end{array}$ & $\begin{array}{l}\text { T. desde } M \text {. } \\
\text { antes } D . C \text {. }\end{array}$ & $\begin{array}{c}\text { T. desde } M \text {. } \\
\text { antes } D \text {. }\end{array}$ \\
\hline 1 & 1 & 25 & O.I.I.A. & $4 \mathrm{~h}: 30 \mathrm{~m}$. & $35 \mathrm{~m}$. & $45 \mathrm{~m}$. \\
\hline 2 & 1 & 21 & O.I.D.P. & $4 \mathrm{~h}: 10 \mathrm{~m}$. & $3 \mathrm{~h}: 10 \mathrm{~m}$. & 4h: $25 \mathrm{~m}$ \\
\hline 5 & 1 & 25 & O.I.D.P. & $22 \mathrm{~h}$ & $3 \mathrm{~h}: 20 \mathrm{~m}$. & Cesárea \\
\hline 6 & 1 & 20 & O.I.I.A. & $21 \mathrm{~h}: 45 \mathrm{~m}$ & $25 \mathrm{~m}$. & $43 \mathrm{~m}$. \\
\hline 9 & 1 & 19 & O.I.I.A. & $15 \mathrm{~h}: 50 \mathrm{~m}$ & $40 \mathrm{~m}$. & $1 \mathrm{~h}$. \\
\hline 10 & 1 & 27 & O.I.D.A. & $25 \mathrm{~h}: 50 \mathrm{~m}$ & $1 \mathrm{~h}: 20 \mathrm{~m}$. & $1 \mathrm{~h}: 40 \mathrm{~m}$ \\
\hline 11 & 1 & 37 & O.I.D.A. & $15 \mathrm{~h}: 30 \mathrm{~m}$ & $6 \mathrm{~h}$. & $7 \mathrm{~h}: 35 \mathrm{~m}$ \\
\hline 12 & 1 & 21 & O.I.I.T. & $11 \mathrm{~h}$ & $2 \mathrm{~h}$. & $2 \mathrm{~h}: 45 \mathrm{~m}$ \\
\hline 13 & 1 & 21 & O.I.D.P. & $11 \mathrm{~h}$ & $1 \mathrm{~h}: 20 \mathrm{~m}$. & $2 \mathrm{~h}: 05 \mathrm{~m}$ \\
\hline 14 & 1 & 25 & S.I.I.A. & $11 \mathrm{~h}: 25 \mathrm{~m}$ & $3 \mathrm{~h}: 05 \mathrm{~m}$. & $4 \mathrm{~h}: 10 \mathrm{~m}$ \\
\hline 16 & 1 & 19 & O.I.I.A. & & $3 \mathrm{~h}: 30 \mathrm{~m}$. & Cesárea \\
\hline 17 & 1 & 16 & O.I.D.P. & $6 \mathrm{~h}$. & $5 \mathrm{~h}: 25 \mathrm{~m}$. & $7 \mathrm{~h}: 40 \mathrm{~m}$ \\
\hline 21 & 1 & 26 & O.I.I.T. & $5 \mathrm{~h}: 15 \mathrm{~m}$. & $4 \mathrm{~h}$ & $4 \mathrm{~h}: 43 \mathrm{~m}$ \\
\hline 22 & 1 & 21 & O.I.D.P. & $5 \mathrm{~h}$. & $4 \mathrm{~h}: 50 \mathrm{~m}$. & $5 \mathrm{~h}: 30 \mathrm{~m}$ \\
\hline 23 & 1 & 22 & O.I.I.T. & $8 \mathrm{~h}$. & $3 \mathrm{~h}: 05 \mathrm{~m}$. & $3 \mathrm{~h}: 30 \mathrm{~m}$ \\
\hline 24 & 1 & 19 & O.I.I.A. & $17 \mathrm{~h}$. & $2 \mathrm{~h}$ & $2 \mathrm{~h}: 25 \mathrm{~m}$ \\
\hline 25 & 1 & 30 & O.I.I.A. & $6 \mathrm{~h}: 15 \mathrm{~m}$ & $1 \mathrm{~h}: 45 \mathrm{~m}$. & $2 \mathrm{~h}: 10 \mathrm{~m}$ \\
\hline 27 & 1 & 20 & O.I.I.P. & $11 \mathrm{~h}: 50 \mathrm{~m}$ & $2 \mathrm{~h}: 10 \mathrm{~m}$. & $2 \mathrm{~h}: 30 \mathrm{~m}$ \\
\hline 30 & 1 & 26 & O.I.I.A. & $7 \mathrm{~h}: 45 \mathrm{~m}$ & $1 \mathrm{~h}: 05 \mathrm{~m}$. & $1 \mathrm{~h}: 43 \mathrm{~m}$ \\
\hline 31 & 1 & 17 & O.I.D.P. & $11 \mathrm{~h}: 30 \mathrm{~m}$ & $2 \mathrm{~h}: 30 \mathrm{~m}$. & $3 \mathrm{~h}: 15 \mathrm{~m}$ \\
\hline 33 & 1 & 20 & O.I.I.A. & $12 \mathrm{~h}: 05 \mathrm{~m}$ & $2 \mathrm{~h}: 25 \mathrm{~m}$. & $6 \mathrm{~h}$. \\
\hline 34 & 1 & 19 & O.I.I.A. & $8 \mathrm{~h}: 30 \mathrm{~m}$. & $55 \mathrm{~m}$. & $1 \mathrm{~h}: 35 \mathrm{~m}$ \\
\hline 35 & 1 & 19 & O.I.T.A. & $13 \mathrm{~h}$. & $2 \mathrm{~h}$ & $2 \mathrm{~h}: 50 \mathrm{~m}$ \\
\hline 37 & 1 & 20 & O.I.I.A. & $14 \mathrm{~h}$. & $1 \mathrm{~h}$. & $1 \mathrm{~h}: 20 \mathrm{~m}$ \\
\hline 38 & 1 & 18 & O.I.I.P. & $4 \mathrm{~h}: 45 \mathrm{~m}$. & $2 \mathrm{~h}: 45 \mathrm{~m}$. & $3 \mathrm{~h}: 38 \mathrm{~m}$ \\
\hline 41 & 1 & 18 & O.I.I.A. & $25 \mathrm{~h}: 50 \mathrm{~m}$ & $2 \mathrm{~h}: 10 \mathrm{~m}$. & $2 \mathrm{~h}: 35 \mathrm{~m}$ \\
\hline 42 & 1 & 19 & O.I.I.A. & $8 \mathrm{~h}: 50 \mathrm{~m}$. & $2 \mathrm{~h}: 30 \mathrm{~m}$. & $3 \mathrm{~h}: 05 \mathrm{~m}$. \\
\hline 43 & 1 & 23 & S.I.I.A. & $10 \mathrm{~h}$ & $40 \mathrm{~m}$. & $48 \mathrm{~m}$. \\
\hline 45 & 1 & 21 & O.I.I.P. & $9 \mathrm{~h}: 55 \mathrm{~m}$. & $5 \mathrm{~h}: 55 \mathrm{~m}$. & Cesárea \\
\hline 48 & 1 & 22 & O.I.D.P. & $5 \mathrm{~h}: 20 \mathrm{~m}$ & $3 \mathrm{~h}: 50 \mathrm{~m}$. & $4 \mathrm{~h}: 40 \mathrm{~m}$ \\
\hline 15 & 2 & 29 & O.I.D.A. & $10 \mathrm{~h}: 35 \mathrm{~m}$ & $1 \mathrm{~h}: 05 \mathrm{~m}$. & $1 \mathrm{~h}: 10 \mathrm{~m}$ \\
\hline 20 & 2 & 18 & O.I.D.P. & $8 \mathrm{~h}: 25 \mathrm{~m}$. & $2 \mathrm{~h}$ & $2 \mathrm{~h}: 45 \mathrm{~m}$ \\
\hline 28 & 2 & 18 & O.I.I.A. & $4 \mathrm{~h}: 25 \mathrm{~m}$. & $50 \mathrm{~m}$. & $1 \mathrm{~h}$. \\
\hline
\end{tabular}




\begin{tabular}{|c|c|c|c|c|c|c|}
\hline $\begin{array}{l}\text { Historia } \\
\text { número }\end{array}$ & Paridad & Edad & V. de P. & $\begin{array}{l}\text { T. de } \mathrm{T} . \\
\text { antes } \mathrm{M} \text {. }\end{array}$ & $\begin{array}{l}\text { T. desde M. } \\
\text { antes } D \text {. C. }\end{array}$ & $\begin{array}{l}\text { T. desde } M \text {. } \\
\text { antes } D .\end{array}$ \\
\hline 29 & 2 & 25 & O.I.I.A. & $5 \mathrm{~h}: 10 \mathrm{~m}$. & $30 \mathrm{~m}$. & $35 \mathrm{~m}$. \\
\hline 36 & 2 & 27 & O.I.I.P. & $3 \mathrm{~h}: 50 \mathrm{~m}$. & $35 \mathrm{~m}$. & $45 \mathrm{~m}$. \\
\hline 39 & 2 & 24 & O.I.I.A. & $5 \mathrm{~h}: 45 \mathrm{~m}$. & $30 \mathrm{~m}$. & $35 \mathrm{~m}$. \\
\hline 44 & 2 & 27 & O.I.I.A. & $10 \mathrm{~h}$. & $30 \mathrm{~m}$. & $35 \mathrm{~m}$. \\
\hline 49 & 2 & 23 & O.I.I.T. & $3 \mathrm{~h}: 45 \mathrm{~m}$. & $1 \mathrm{~h}: 05 \mathrm{~m}$. & $1 \mathrm{~h}: 15 \mathrm{~m}$. \\
\hline 50 & 2 & 17 & O.I.I.A. & $12 \mathrm{~h}: 20 \mathrm{~m}$ & $1 \mathrm{~h}: 05 \mathrm{~m}$. & $1 \mathrm{~h}: 15 \mathrm{~m}$. \\
\hline 3 & 8 & 33 & O.I.I.A. & $4 \mathrm{~h}: 05 \mathrm{~m}$. & $45 \mathrm{~m}$. & $48 \mathrm{~m}$. \\
\hline 4 & 5 & 29 & O.I.I.A. & $7 \mathrm{~h}$. & $40 \mathrm{~m}$. & $45 \mathrm{~m}$. \\
\hline 7 & 6 & 29 & O.I.I.A. & $3 \mathrm{~h}$. & $35 \mathrm{~m}$. & $40 \mathrm{~m}$ \\
\hline 8 & 3 & 32 & O.I.D.T. & $4 \mathrm{~h}: 25 \mathrm{~m}$. & 1h: $35 \mathrm{~m}$. & $2 \mathrm{~h}$. \\
\hline 18 & 4 & 25 & O.I.I.A. & $6 \mathrm{~h}$. & $40 \mathrm{~m}$. & $45 \mathrm{~m}$ \\
\hline 19 & 5 & 22 & O.I.I.A. & $8 \mathrm{~h}: 15 \mathrm{~m}$. & $30 \mathrm{~m}$. & $40 \mathrm{~m}$ \\
\hline 26 & 3 & 17 & O.I.I.A. & $4 \mathrm{~h}: 05 \mathrm{~m}$. & $1 \mathrm{~h}: 05 \mathrm{~m}$. & $1 \mathrm{~h}: 15 \mathrm{~m}$. \\
\hline 32 & 3 & 24 & O.I.I.A. & $5 \mathrm{~h}: 40 \mathrm{~m}$. & $1 \mathrm{~h}: 15 \mathrm{~m}$. & $1 \mathrm{~h}: 20 \mathrm{~m}$ \\
\hline 40 & 8 & 42 & O.I.I.T. & $14 \mathrm{~h}: 20 \mathrm{~m}$ & $25 \mathrm{~m}$. & $30 \mathrm{~m}$ \\
\hline 46 & 4 & 24 & O.I.D.T. & $4 \mathrm{~h}: 35 \mathrm{~m}$. & $1 \mathrm{~h}: 45 \mathrm{~m}$. & $2 \mathrm{~h}: 10 \mathrm{~m}$ \\
\hline 47 & 7 & 33 & O.I.I.P. & $11 \mathrm{~h}: 20 \mathrm{~m}$ & $30 \mathrm{~m}$. & $43 \mathrm{~m}$. \\
\hline \multirow[t]{2}{*}{51} & 4 & 24 & O.I.I.A. & $5 \mathrm{~h}: 40 \mathrm{~m}$. & $35 \mathrm{~m}$. & $40 \mathrm{~m}$ \\
\hline & & CASOS & TRATADOS & SOLO CON & AMNIOTOMIA & \\
\hline 1 & 2 & & O.I.I.A. & & $3 \mathrm{~h}: 55 \mathrm{~m}$. & $4 \mathrm{~h}: 10 \mathrm{~m}$ \\
\hline 2 & 2 & & O.I.D.P. & & $5 \mathrm{~h}$. & $5 \mathrm{~h}: 50 \mathrm{~m}$. \\
\hline 3 & 5 & & O.I.I.A. & & $4 \mathrm{~h}: 30 \mathrm{~m}$ & $4 \mathrm{~h}: 55 \mathrm{~m}$ \\
\hline 4 & 2 & & O.I.D.P. & & $4 \mathrm{~h}: 30 \mathrm{~m}$. & $4 \mathrm{~h}: 55 \mathrm{~m}$. \\
\hline 5 & 1 & & O.I.I.A. & & $7 \mathrm{~h}$. & $7 \mathrm{~h}: 38 \mathrm{~m}$ \\
\hline 6 & 1 & & O.I.D.P. & & $18 \mathrm{~h}: 40 \mathrm{~m}$. & $19 \mathrm{~h}: 40 \mathrm{~m}$ \\
\hline 7 & 6 & & O.I.D.P. & & $11 \mathrm{~h}$. & $11 \mathrm{~h}: 45 \mathrm{~m}$ \\
\hline 8 & 3 & & O.I.I.A. & & $4 \mathrm{~h}: 55 \mathrm{~m}$. & $5 \mathrm{~h}: 10 \mathrm{~m}$ \\
\hline 9 & 2 & & O.I.I.A. & & 6h: $5 \mathrm{~m}$. & $6 \mathrm{~h}: 10 \mathrm{~m}$ \\
\hline 10 & 1 & & O.I.I.A. & & $8 \mathrm{~h}$. & $9 \mathrm{~h}$. \\
\hline
\end{tabular}

CONVENCIONALES: V. de P.: variedad de posición.

T. de T. antes M.: tiempo de trabajo antes de la medicación.

T. desde M. antes D. C.: tiempo transcurrido desde la medicación hasta dilatación completa. 


\section{Comentario}

A pesar de haber sistematizado el plan de tratamiento, con aplicación de ocitócicos y amniotomía para todos los casos, en diecisiete no se aplicaron ocitócicos, por existir contracciones uterinas de suficiente intensidad y frecuencia, y aun en algunos verdadera polisistolia.

De los casos en los cuales no se llevó a cabo la amniotomía al iniciar la medicación, en diez casos no se llegó a hacer, por haber ingresado las pacientes con membranas rotas, seis de ellas primigestantes, entre las cuales una terminó su caso en cesárea transperitoneal (16 horas) y otra con parto con feto muerto y macerado (19 horas). En nueve pacientes, la amniotomía no se practicó inicialmente, habiendo dejado que se rompieran las membranas espontáneamente, una vez completada la dilatación, - bien llevando a cabo la amniotomía durante el segundo período del parto (casos $41,42,43,44,45,46,47,48,49,50$ y 51). En estos últimos casos, no se observó alargamiento del período de dilatación en relación con los demás casos, habiendo obtenido un máximo de 4 horas con 45 minutos (caso 45); y un mínimum de 30 minutos (caso 44 ).

En relación con las dosis de medicamento utilizadas, en el $47 \%$ (24 casos) de los casos tratados, la dosis de un gramo fue suficiente para obtener una dilatación completa. El 35,2\% fueron tratados con 2 gramos de O. T. P. (18 casos), varios de los cuales no hubieran requerido más de un gramo (historias: 15, 24, $26,32,33,49)$, por encontrarse en más de 7 centímetros al aplicar la segunda dosis; el resto fueron, sin duda, casos de hipertonia (historias: $5,11,12,13,25,27,31,35,38,41,42,45$ ).

Dosis de tres gramos fueron administradas en el $17,6 \%$ (9 casos) del total de historias; entre éstos, solamente hay un caso cle hipertonia que requirió una dosis más elevada (caso 22), el resto, son casos en los cuales la dosis mínima útil hubiera podicio ser menor (historias: 2, 14, 16, 17, 20, 21, 23, 48).

Como anteriormente se hizo notar, en la estadística figuran tres historias, en las cuales el caso se resolvió por cesárea. Es necesario insistir que tratándose de valorar la utilidad de la droga en el primer período del parto, la inclusión de dichos casos, no hace más que reafirmar su utilidad, al lograr pruebas de trabajo más cortas, más efectivas (al descontar el factor distocia dinámica), y por lo tanto más seguras tanto para la madre como para el feto. 


\section{SUMARIO}

Preséntanse sesenta y un casos obstétricos estudiados en la Clínica Primero de Mayo de Bogotá, de los cuales 51 se trataron en el primer período del parto con O. T. P. y 10 solamente con amniotomía.

1. La bondad de la droga como abreviante del trabajo del parto, fue comprobada en el presente estudio.

2. La ausencia de efectos nocivos en dosis terapéuticas, tanto para la madre como para el hijo, al administrar la droga por via oral, han quedado demostradas.

3. Las cifras que aparecen en la sección de resultados del presente trabajo atestiguan la gran utilidad de la droga en el primer periodo del parto, disminuyendo la duración de éste.

4. La administración de la droga aun sin practicar precozmente la ruptura de las membranas (amniotomía), y sin el empleo de ocitócicos, obtiene la disminución marcada en la duración del primer período.

Todos los fetos de los casos tratados nacieron vivos y lloraron al nacer, a excepción de un caso en el que la madre llegó a la clínica con auscultación y movimientos fetales negativos ciesde hacía algún tiempo, y en el cual se obtuvo un feto muerto y macerado.

Tanto el alumbramiento como el puerperio evolucionaron dentro de lo normal en todos los casos.

En los 10 casos en los que únicamente se practicó la amniotomía sin haber administrado la droga, tanto el período de dilatación como el período expulsivo se hicieron comparativamente más demorados.

Queda demostrada la poca influencia de la amniotomía en la evolución del trabajo del parto, al no acelerarse éste con su práctica, ni tampoco demorarse, en los casos en los cuales fue omitida hasta completarse la dilatación, bajo la acción de la droga en experimentación.

La dosis de un gramo de 3--Orto, Toloxi-1, 2-Propanodiol (2 comp. de 0,50), demostró ser en la mayoría de los casos $(47 \%)$, suficiente para obtener resultados satisfactorios. 


\section{BIBLIOGRAFIA}

1. BERGER F. M. y BRADLEY W.-The pharmacologic properties of Myanesin. Brit. J. Pharmacol. I: 265-272. (Dic. 1946).

2. SARDIÑAS R. ALFREDO.-Método original para abreviar el tiempo de la duración del parto. La Habana, Cuba. Nov. de 1954. 3-5-6-8-10.

3. BERGER F. M. LANCET.-I: 97 (1947).

4. GONZALEZ M. EFRAIM.-Algunas observaciones sobre el Tolserol en el parto. Tesis de grado. 1953. Bogotá. 21-23.

5. FORERO VELEZ RICARDO.-Conferencias de Obstetricia tomadas en 1953, no revisadas.

6. MORAGUES JAIME.-Tratado de Clínica Obstétrica. Buenos Aires, Argentina. 1950. 120-126.

7. GIBELI GASTON.-“Acción de un Simpaticolítico (dihidroergotamina), sobre la dinámica uterina". "Revista de Obstetricia y Ginecología Latinoamericanas". Número 8. Agosto de 1955. Argentina. 327-330.

8. CALDEYRO-BARCIA, R.; ALVAREZ, H.; POSEIRO, J. J.-Contractilidad uterina normal y anormal en el parto. Revista Triángulo. Vol. II, número 2. Septiembre de 1955. Basilea, Suiza. 41-51.

9. DAVISON, M. H. ARMSTRONG.-Haemolitic effects of Myanesin. Lancet 257: 110 (Dec. 10. 1949).

10. HENNEMAN E., and SCHERRER, JEAN.-The mode of action ef Myanesin on the central nervous system.

Tr. Am. Meurol. A. 74. 208-211 (1949).

11. COWEN, J.-Toxin effects of Myanesin.

Lancet. 254:865-966 (junio 19, 1948).

12. NUBIOLA y ZARATE.-Tratado de Obstetricia, 1951. España. Tomo I. Páginas 350-386, y de 171-172.

13. DEXEUS FONT, S.-Tratado de Obstetricia, 1949, España. Tomo I. Páginas 222-258.

14. DE LEE - GREENHILL.-Principios y Práctica de Obstetricia. 1947, México. Tomo I. Páginas: 178-203.

15. DAVIS CARL HENRY.-Gynecology and Obstetrics. 1945. Hagerstown Maryland. Vol. I. Chapter VI.

16. BECK ALFRED.-Obstetrical Practice. 1947. Fourth Edition. Baltimore. Chapter XII.

17. DUBRISAY L. et Jeannin C.-Précis d' Accouchement. $8^{\text {a }}$ Edition, París. 1936. Páginas: 123 a 149.

18. STANDER HENRICUS J.-Obstetricia (Williams). $7^{\text {a }}$ Edición. México. Páginas 72 a 74.

19. EASTMAN NICHOLSON J.-Obstetricia de Williams. $2^{\text {a }}$ Edición en Español. México. Páginas: 45-46.

20. CONIL MONTOBBIO VICTOR.-Tratado de Ginecología. 2a Edición. 1950. España. Páginas: 41-46. 